\title{
Validación del Cuestionario de Maltrato entre Iguales por Abuso de Poder (MIAP) para escolares
}

\author{
FELIPE LECANNELIER ${ }^{\mathrm{a}}$, JORGE VARELA ${ }^{\mathrm{a}}$, JORGE RODRÍGUEZ ${ }^{\mathrm{b}}$, \\ MARIANELA HOFFMANN ${ }^{a}$, FERNANDA FLORES ${ }^{a}$, LORENA ASCANIO ${ }^{a}$
}

Centro de Estudios

Evolutivos e Intervención en el Niño, Facultad de Psicología, Universidad del Desarrollo. asicólogo. ${ }^{\mathrm{b}}$ Estadístico

Recibido el 13 de mayo de 2010, acepado el 4 de marzo de 2011

Correspondencia: Felipe Lecannelier Av. Las Condes 12.438, Lo Barnechea

Fax: (56 2) 3279281 E-mail: flecannelier@udd.cl

\section{A self administered survey to assess bullying in schools}

Background: Bullying is common in schools and has negative consequences. It can be assessed using a self-reported instrument. Aim: To validate a Spanish self-reporting tool called "Survey of High School Bullying Abuse of Power" (MIAP). Material and Methods: The instrument has 13 questions, of which 7 are multiple choice, rendering a total of 49 items. It was applied to 2.341 children of seventh and eighth grade attending private, subsidized and municipal schools in the city of Concepcion, Chile. Expert judge analysis and estimated reliability using the Cronbach Alpha were used to validate the survey. Results: The instrument obtained a Cronbach Alpha coefficient of 0.8892, classified as good. This analysis generated four scales that explained 30.9\% of the variance. They were called "Witness Bullying" with 18 items, accounting for $11.4 \%$ of the variance, "Bullying Victim" with 12 items, accounting for $7.5 \%$ of the variance, "Bullying Perpetrator and Severe bullying Victim", with 10 items explaining $6.4 \%$ of the variance and "Aggressor Bullying" with 6 items accounting for 5.7\% of the variance. Conclusions: The MIAP can recognize four basic factors that facilitate the analysis and understanding of bullying, with good levels of reliability and validity. The remaining questions also deliver valuable information.

(Rev Med Chile 2011; 139: 474-479).

Key words: Bullying; Questionnaires; Schools.
$\mathrm{E}$ 1 presente artículo describe la validación de un instrumento adaptado en Chile para medir el acoso escolar entre pares. Pretende identificar factores explicativos del fenómeno que facilite su análisis y comprensión, oriente planes de acción, prevención e intervención, sirva de insumo para evaluar estrategias de intervención y contribuya en la generación de mayor conocimiento científico.

Hoy existen investigaciones llevadas a cabo en varias partes del mundo que permiten conocer más sobre diversas manifestaciones y prevalencias del fenómeno. No obstante, Chile tiene menos recorrido de investigación en esto. Estudios internacionales han comparado datos sobre acoso escolar (bullying) el cual se sitúa en un rango desde 7-8\% (Suecia) hasta 40-50\% (Lituania) ${ }^{1}$. En Chile, cuando se habla de este fenómeno, $10,7 \%$ de estudiantes se declaran víctimas de amenazas permanentes y discriminación por parte de sus pares ${ }^{2}$. Se sabe que los hombres suelen ser más violentos físicamente que las mujeres, pero ellas suelen utilizar más la violencia relacional ${ }^{3}$, o usar la agresión indirecta, pero no de forma exclusiva, por tanto, no se puede generalizar automáticamente el tipo de agresión al género ${ }^{4}$. La conducta de acoso escolar 
suele empezar de un modo más perceptible a los 6-7 años, llega a su peak a los 10-13 años y suele disminuir en la adolescencia. También estudios actuales evidencian conductas de intimidación física en la desde la edad preescolar, donde niños victimizados con pocos amigos son más vulnerables a sufrir futuras situaciones de agresión ${ }^{5}$; e incluso que el nivel más alto de agresión física se encuentra durante los primeros 24 y 42 meses de vida, "aprendiendo" posteriormente, en la gran mayoría de los casos, a regular dicha agresión ${ }^{6}$.

Los niveles de prevalencia de la violencia están determinados por los constructos y aproximaciones metodológicas que se usen. En este sentido, es posible reconocer dos grandes líneas de comprensión y análisis del fenómeno ${ }^{7}$. Una mirada ha estado centrada en definir el acoso escolar (bullying) basada en los trabajos de Dan Olweus ${ }^{8}$, entendida como un tipo de agresión negativa e intencional hacia una víctima de forma repetida durante un tiempo, en una posición de desbalance de fuerzas y poder, donde la víctima no puede defenderse. Las aproximaciones metodológicas del acoso escolar provienen de dos fuentes. Observaciones indirectas por medio de la nominación de docentes o de pares, o métodos de observación directa. El método más común son cuestionarios aplicados directamente a los estudiantes, ya sea identificando víctimas y victimarios por medio de autorreportes, o cuestionarios basados en nominaciones de pares.

La otra línea de investigación usa la nomenclatura de victimización en general, la cual estudia agresiones de diversa índole (físicas, psicológicas, etc) y direcciones, pero no está presente la repetición de la agresión ni el desbalance de poder. Para estudiar la victimización existen dos grandes fuentes de información. Una consiste en revisar los registros policiales sobre estas situaciones puntuales de violencia, pero habitualmente esconden una importante cifra negra. Una segunda fuente son las mediciones de auto-reporte a víctimas o agresores. Esta última fuente es la más utilizada para medir estos fenómenos ${ }^{10}$.

Otros autores señalan que es importante tener en cuenta varios tipos de instrumentos o fuentes de información que permitan identificar a las víctimas, agresores y victimarios ${ }^{11}$. Tales métodos incluyen: Escalas y encuestas de auto reporte, nominación de pares, nominación por parte de los docentes y observación directa de la conducta.
Las escalas y encuestas de autorreporte son las más usadas por los investigadores y por los establecimientos educacionales para reunir información sobre el acoso escolar. Una escala tipo pregunta a los estudiantes de forma directa -pero anónima asegurando la confidencialidad de los datos-cuán seguido ha estado involucrado en determinados comportamiento en un rango de tiempo determinado (por ejemplo en los últimos 30 días), lo cual permite ir construyendo las determinadas escalas. Otros instrumentos, dan una definición de acoso escolar, y después se pregunta sobre dichas situaciones de victimización. La nominación de pares o profesores es usada para diseñar intervenciones focalizadas con estudiantes. No es una medición masiva, sino que busca identificar algunos alumnos que puedan ser identificados por sus pares o profesores en una situación de mayor riesgo. La observación directa en los contextos naturales de los estudiantes es una buena técnica para recolectar información sobre la frecuencia y los roles de los estudiantes en el acoso escolar. Esta metodología provee una invaluable información acerca de la interacción de los estudiantes, pero requiere de una larga data de mediciones y de variados escenarios (sala, patios, casino, etc) para poder entregar mayor información del fenómeno.

En Chile, el fenómeno de violencia y acoso escolar se ha empezado a estudiar con mayor precisión en los últimos años ${ }^{12}$. Inicialmente, se incorporaron preguntas sobre violencia en cuestionarios de aplicación masiva tales la encuesta del Consejo Nacional para el Control de Estupefacientes $^{13}$ o del Instituto Nacional de la Juventud ${ }^{14}$. Dentro de los trabajos pioneros se pueden destacar las investigaciones de la Universidad del Desarrollo (Lecannelier F. Un proyecto Bullying para la detección e intervención de la violencia escolar entre pares en Chile. Memorias de las XII Jornadas de Investigación, Universidad de Buenos Aires, 2005) y otras investigaciones de corte cualitativo ${ }^{15}$, que fueron marcando una pauta y relevaron el fenómeno desde el mundo académico y científico. Desde las políticas públicas nacionales se ha empezado a medir el fenómeno de la violencia escolar desde el año 2005, con una encuesta de representatividad nacional tipo autorreporte aplicada a los estudiantes y profesores, incorporando en su última versión a los asistentes de la educación. Sin embargo, no existe una encuesta que mida con mayor precisión el acoso escolar, de fácil y corta aplicación, que 
permita conocer aspectos relevantes del fenómeno y sea fácilmente administrable por los colegios.

El acoso escolar está determinado por la interacción de tres actores: agresor, víctima y un observador, en el contexto escolar, compartiendo en algunas ocasiones más de uno de esos roles ${ }^{16}$. Por lo mismo, los instrumentos de evaluación del fenómeno deben ser capaces de revelar información sobre todos estos protagonistas, el que comete la agresión, el que la recibe y el que observa, junto con otros aspectos socioafectivos del grupo de pares $^{17}$. En este sentido, el objetivo final del presente estudio es poder validar el MIAP en la realidad nacional identificando a la base factores que estén a la base del instrumento, que expliquen de forma robusta el fenómeno.

\section{Material y Método}

El método es de tipo metodológico y descriptivo con diseño no experimental transversal, realizándose la evaluación en un solo momento, a partir de una muestra escolar dirigida a los establecimientos que accedieron a participar. Esta decisión se tomó de acuerdo al interés expresado por los colegios por evaluar la frecuencia de acoso escolar en su comunidad escolar.

El estudio fue presentado al comité de ética de la Facultad de Psicología de la Universidad del Desarrollo. Respecto del consentimiento informado, los directores de los colegios participantes informaron a los padres del estudio, haciéndose responsables de la importancia de tener esa información, solicitando la respectiva autorización.

\section{Instrumentos}

El cuestionario aplicado en Chile es una adaptación del instrumento denominado "Cuestionario de Secundaria de Maltrato entre Iguales por Abuso de Poder" (MIAP) ${ }^{18}$. Es un tipo de instrumento de autorreporte completado por los mismos estudiantes. Contiene 13 preguntas, de las cuales 7 son de selección múltiples con una sola respuesta posible ( $\mathrm{N}^{\circ} 1,2,3,4,6,8$ y 10$)$, con un total de 49 ítems, todas ellas de carácter ordinal. Las preguntas restantes son de tipo dicotómica o que pueden responder más de una alternativa de respuesta $\left(\mathrm{N}^{\circ} 5,7,9,11,12\right.$ y 13$)$. Por lo tanto, la adaptación del instrumento se aplicó sólo a las preguntas ordinales con una sola respuesta posible.

\section{Procedimiento}

Se llevó a cabo una validación de contenido del instrumento, la cual consistió en trabajar con 3 jueces expertos, los cuales podían sugerir, modificar, eliminar o agregar algún ítem. La eliminación de los ítems se aplicó cuando al menos 2 de los jueces hacían algún reparo en el mismo ítem, ya sea eliminar, agregar o modificar.

El procedimiento de recolección de datos se realizó sobre la base de contactos directos a colegios representativos de la ciudad de Concepción (municipalizados, subvencionados y particulares). Una vez realizado el contacto, un equipo de psicólogos acudió a cada colegio, previa coordinación con el profesor jefe del curso. Los psicólogos explicaron a los alumnos el cuestionario, remarcando su carácter anónimo y la posibilidad de realizar preguntas.

Una vez obtenida la información se ingresaron los datos al programa SPSS, versión 17, donde se realizó un análisis descriptivo resumiendo la información en indicadores de tendencia central y de dispersión. Para la estimación de la confiabilidad se aplicó el Alpha de Cronbach y en base a los resultados, se realizó un análisis de validación a través del análisis factorial con varianza máxima.

\section{Resultados}

\section{Participantes}

La muestra del estudio fue por conveniencia. La población estuvo constituida por escolares pertenecientes a la ciudad de Concepción que cursaban entre séptimo y octavo básico y que estuvieron presentes el día de la aplicación del instrumento. Todos los colegios invitados a participar lo hicieron. Así la muestra final fue de 6 colegios subvencionados, 6 particulares y 5 municipalizados. El tamaño muestral fue de 2.341 escolares entre 11 y 17 años. La edad promedio fue 13 años y desviación estándar $\pm 0,8$ años, con ambos géneros. Cincuenta y tres coma tres porciento eran hombres, quienes tuvieron una edad promedio de 13,1 años; las mujeres, por su parte, con 12,9 años. La variabilidad fue también muy similar en ambos grupos, con 0,8 y 0,7 años respectivamente.

En los resultados obtenidos en el proceso de validación de contenidos de los tres jueces, no se realizó ningún cambio de ítems respecto al instrumento original.

El proceso de validación generó cuatro escalas 
que explican el 30,9\% de la varianza. Se denominaron "Testigo Bullying" -1-, con 18 ítems, que explica el 11,4\%, "Víctima Bullying" -2- con 12 ítems que explican 7,5\%, "Agresor y Víctima Bullying Grave" -3- de 10 ítems que explican 6,4\% y "Agresor Bullying" -4- con 9 ítems que explican $5,7 \%$ de la varianza respectivamente (Tabla 1), de tal manera, que cada escala quedó conformado de la siguiente manera (Tabla 2).

En la Tabla 3, se observa que los estudiantes en las escalas Testigo Bullying, Víctima Bullying y Agresor y Víctima Bullying Grave tienen valores menores a los posibles, en cambio la escala Agresor Bullying obtuvo ambos valores teóricos extremos.

Las escalas de Agresor y Víctima de Bullying grave y Agresor Bullying tienen valores más homogéneos que los otros dos constructos que tienen puntajes más variables. Como se observa en la Tabla 4.

Como parte de la validación de constructos se realizó una estimación de confiabilidad. En los 49 ítems observados se obtuvo un coeficiente de Alpha de Cronbach de 0,8892, clasificado como bueno. Las escalas de Testigo Bullying la conforman 18 ítems, con una confiabilidad de 0,8559 considerado buena. La escala denominada Víctima Bullying la conforman 12 ítems, con un valor de Alpha de Cronbach de 0,8029 considerado bueno. La escala Agresor y Víctima de Bullying Grave contiene 10 ítems con una confiabilidad de 0,7451 y finalmente, la escala Agresor Bullying la conforman 9 ítems con un valor de Alpha de Cronbach 0,7219, que también puede ser considerada como buena confiabilidad (Tabla 5).

Tabla 1. Composición de la varianza explicada por los escalas autovalores iniciales y saturaciones cuadrado de la rotación

\begin{tabular}{|ccccccc|}
\hline Escalas & Total & \% de la varianza & \% acumulado & Total & \% de la varianza & \% acumulado \\
\hline 1 & 8,049 & 16,426 & 16,426 & 5,550 & 11,4 & 11,326 \\
\hline 2 & 2,699 & 5,508 & 21,933 & 3,680 & 7,5 & 18,836 \\
\hline 3 & 2,396 & 4,891 & 26,824 & 3,143 & 6,4 & 25,249 \\
4 & 2,037 & 4,158 & 30,982 & 2,809 & 5,7 & 30,982 \\
\hline
\end{tabular}

Tabla 2. Distribución de los ítems en las escalas

\begin{tabular}{|ll|}
\hline Escalas & Ítems \\
\hline Testigo bullying & $1: \mathrm{a}, \mathrm{b}, \mathrm{c}, \mathrm{d}, \mathrm{e}, \mathrm{f}, \mathrm{g}, \mathrm{h}, \mathrm{i}, \mathrm{j}, \mathrm{k}, \mathrm{I}$ y $\mathrm{m} ; 2 \mathrm{a}, 2 \mathrm{~b}, 3 \mathrm{a}, 3 \mathrm{~b}, 3 \mathrm{c}$ \\
\hline Víctima bullying & $4,6,8 \mathrm{a}, 8 \mathrm{~b}, 8 \mathrm{c}, 8 \mathrm{~d}, 8 \mathrm{e}, 8 \mathrm{f}, 8 \mathrm{~g}, 8 \mathrm{~h}, 8 \mathrm{i}, 8 \mathrm{j}$ \\
\hline Agresor y víctima bullying grave & $8 \mathrm{k}, 8 \mathrm{l}, 8 \mathrm{~m}, 8 \mathrm{n}, 8 \mathrm{o}, 10 \mathrm{~g}, 10 \mathrm{~h}, 10 \mathrm{l}, 10 \mathrm{~m}, 10 \mathrm{n}$ \\
\hline Agresor bullying & $10 \mathrm{a}, 10 \mathrm{~b}, 10 \mathrm{c}, 10 \mathrm{~d}, 10 \mathrm{e}, 10 \mathrm{f}, 10 \mathrm{i}, 10 \mathrm{j}, 10 \mathrm{k}$ \\
\hline
\end{tabular}

Tabla 3. Valores mínimos y máximo teóricos y observados por escalas

\begin{tabular}{|lcccc|}
\hline Escalas & \multicolumn{2}{c}{ Teórico } & \multicolumn{2}{c|}{ Observado } \\
& Mínimo & Máximo & Mínimo & Máximo \\
\hline Testigo bullying & 18 & 72 & 19 & 69 \\
Víctima bullying & 12 & 49 & 12 & 44 \\
\hline Agresor víctima bullying grave & 13 & 52 & 13 & 47 \\
\hline Agresor bullying & 6 & 24 & 6 & 24 \\
\hline
\end{tabular}


Tabla 4. Indicadores descriptivos de las escalas

\begin{tabular}{|lcccc|}
\hline Escalas & Media & Desv. típ & Inferior & Superior \\
\hline Testigo bullying & 36,7467 & 7,76411 & 36,4232 & 37,0702 \\
\hline Víctima bullying & 17,8161 & 4,46003 & 17,6322 & 18,0000 \\
\hline Agresor y víctima bullying grave & 10,6430 & 1,80872 & 10,5690 & 10,7170 \\
Agresor bullying & 12,2863 & 2,82076 & 12,1144 & 12,4582 \\
\hline
\end{tabular}

Tabla 5. Distribución de las confiabilidad de los escalas

\begin{tabular}{|lccl|}
\hline Escalas & Correlación & n de ítem & ítem \\
\hline Testigo bullying & 0,8559 & 18 & $1: \mathrm{a}, \mathrm{b}, \mathrm{c}, \mathrm{d}, \mathrm{e}, \mathrm{f}, \mathrm{g}, \mathrm{h}, \mathrm{i}, \mathrm{j}, \mathrm{k}, \mathrm{I}$ y m; 2a, 2b, 3a, 3b, 3c \\
\hline Víctima bullying & 0,8029 & 12 & $4,6,8 \mathrm{a}, 8 \mathrm{~b}, 8 \mathrm{c}, 8 \mathrm{~d}, 8 \mathrm{e}, 8 \mathrm{f}, 8 \mathrm{~g}, 8 \mathrm{~h}, 8 \mathrm{i}, 8 \mathrm{j}$ \\
\hline Agresor víctima bullying grave & 0,7451 & 10 & $8 \mathrm{k}, 8 \mathrm{l}, 8 \mathrm{~m}, 8 \mathrm{n}, 8 \mathrm{o}, 10 \mathrm{~g}, 10 \mathrm{~h}, 10 \mathrm{l}, 10 \mathrm{~m}, 10 \mathrm{n}$ \\
\hline Agresor bullying & 0,7219 & 9 & $10 \mathrm{a}, 10 \mathrm{~b}, 10 \mathrm{c}, 10 \mathrm{~d}, 10 \mathrm{e}, 10 \mathrm{f}, 10 \mathrm{i}, 10 \mathrm{j}, 10 \mathrm{k}$ \\
\hline
\end{tabular}

Tal como se señaló el análisis de validación se llevó a cabo con las preguntas múltiples con una sola respuesta posible. En las preguntas restantes se observan algunos resultados de interés en algunas variables, tales como, 63,6\% de los escolares señaló que se había unido a un grupo o a otro compañero para molestar a alguien en forma continua desde que comenzó. Uno de cada tres escolares habló con su familia o profesor sobre la situación de maltrato. En cuanto a la actitud de los compañeros de curso cuando los estudiantes reportan molestar a otro compañero (victimario)*, la mayoría no hacía nada $(52,5 \%)$,

\section{Discusión}

El objetivo del artículo era dar cuenta la validación de un instrumento que midiera acoso escolar (bullying) en la realidad nacional teniendo en cuenta diferentes aspectos del fenómeno. De esta forma, el instrumento utilizado originalmente en España, y ahora en Chile, presentó buenos niveles de confiabilidad en la realidad nacional creando cuatro escalas de análisis: Testigo Bullying, Víctima Bullying, Agresor Bullying y Agresor-Víctima
Bullying. Cada una de ellas permitió conocer con profundidad el reporte de los estudiantes desde diferentes roles en el círculo de la victimización, confirmando la idea sobre la existencia de distintos roles en torno al fenómeno. De la misma forma, entrega información resumida y agrupada según la posición que se reporta. En relación con esto, resulta interesante el factor que reporta el fenómeno desde una posición de víctima y victimario, reforzando la tesis sobre la posible alternancia de roles y su posición menos estática en torno a la victimización.

A su vez, la validación del instrumento permite hacer uso de todos los ítems, ya que las preguntan que no formaron parte del análisis de confiabilidad, pueden ser utilizadas por separado y entregan también información relevante y de corte más cualitativo para el estudio del fenómeno.

La validación descrita puede ser usada en la realidad nacional y presenta la ventaja de ser de fácil aplicación y posterior análisis de cada una de las escalas creadas y de las preguntas del análisis cualitativo.

A pesar de contar con un buen tamaño muestral, una limitación del estudio radica la realización del mismo en una comuna del país, lo cual no ne-

${ }^{\star}$ El enunciado de la pregunta fue: Cuando tú molestas a alguien en forma continua, ¿Qué hacen tus compañeros? 
cesariamente es reflejo para las restantes. Se recomienda a futuro, seguir analizando el instrumento, fortaleciendo sus valores de confiabilidad. Además, si bien presentó buenos niveles de confiabilidad, no fue posible comparar con otra medición del fenómeno, ya que fue una de las aplicaciones pioneras del fenómeno en el país.

Agradecimientos: Los autores agradecen a todos los colegios de la ciudad de Concepción por haber participado de este estudio. También a la Universidad del Desarrollo por fomentar estos espacios de investigación.

\section{Referencias}

1. Nansel T, Craig W, Overpeck MD, Saluja G, Ruan J, \& Health Behavior in School-aged Children Bullying Analyses Working Group. Cross-national consistency in the relationship between bullying behaviors and psychosocial adjustment. Arch Pediatr Adolesc Med 2004; 158: $730-6$.

2. Ministerio del Interior. Chile. División de Seguridad Pública 2008. Disponible en http://www.seguridadciudadana.gob.cl/estudiosyencuestas.html [Consultado el 20 de diciembre de 2008].

3. Pellegrini AD, Long JD. A longitudinal study of bullying, dominance, and victimization during the transition from primary school to secondary school. British Journal of Developmental Psychology 2002; 20: 259-80.

4. Salmivalli C, Kaukiainen A. "Female aggression" revisited: Variable- and person-centered approaches to studying gender differences in different types of aggression. Aggress Behav 2004; 30: 158-63.

5. Perren S, Alsaker F. Social behavior and peer relationship of victims, bullyi-victims, and bullies in Kindergarten. J Child Psychol Psychiatry 2006; 47: 45-57.

6. Tremblay R. Decade of Behavior distinguished lecture: Development of physical aggression during infancy.
Infant Mental Health Journal 2004; 25: 399-407.

7. Lucas B, Pulido R, Martín G, Calderón S. Violencia entre iguales en educación primaria: un instrumento para su evaluación. Psicología Educativa 2008; 14: 47-62.

8. Olweus D. Bullying at school: What we know and what we can do. Cambridge Blackwell Publishers, 1993.

9. Cerezo F, Ato M. Bullying in Spanish and English pupils: A sociometric perspective using the BULLS questionnaire. Educational Psychology 2005; 25: 353-67.

10. Elliott D, Hamburg B, Williams,K. Violence in American schools: an overview. En Elliott D, Hamburg B, Williams K. (ed) Violence in American Schools. Cambridge University Press, 1998.

11. Espelage D, Swearer S. Research on School Bullying and Victimization: What Have We Learned and Where Do We Go from Here? School Psychology Review 2003; 32: 365-83.

12. Madriaza P. Violencia escolar en Chile. En Guajardo, C. Seguridad y Prevención: La situación en Argentina, Chile y Uruguay durante 2007. Informe de análisis comparativo. Centro Internacional para la Prevención de la Criminalidad, 2008.

13. CONACE (2006). Sexto Estudio Nacional de Drogas en Población Escolar de Chile, 2005. $8^{\circ}$ Básico a $4^{\circ}$ Medio. Informe Final. CONACE. Ministerio de Interior, Gobierno de Chile.

14. INJUV. Tercera encuesta nacional de Juventud. Instituto Nacional de la Juventud, Gobierno de Chile, 2000.

15. García M, Madriaza P. Sentido y sinsentido de la violencia escolar: Análisis cualitativo del discurso de estudiantes chilenos. Psykhe 2005; 14: 165-80.

16. Solberg M, Olweus D, Endresen I. Bullies and victims at school: Are they the same pupils? British Journal of Educational Psychology 2007; 77: 441-64.

17. Salmivalli C, Lagerspetz K, Björkqvist K, Österman K, Kaukialnen A. Bullying as a Group Process: Participant Roles and Their Relations to Social Status Within the Group. Aggress Behav 1996; 22: 1-15.

18. Defensor del Pueblo 2007. Disponible en www.defensordelpueblo.es [Consultado el 20 de diciembre de 2009]. 